\title{
The most important and influential papers in tobacco control: results of an online poll
}

\section{S Chapman}

Tobacco Control 2005;14:e1 (http://www.tobaccocontrol.com/cgi/content/full/14/5/el). doi: 10.1136/tc.2005.013177

a a recent issue, we published a list of the 100 most cited authors who have published work relevant to tobacco control. ${ }^{1}$ We also listed the 50 most cited papers in our field. That exercise produced lists of authors and papers dominated by "big epi" work: papers mainly establishing the contribution of tobacco use to disease. Such papers are often published in high impact factor journals and tend to be cited in the introductory sections of other papers.

Constructing the lists by citations alone resulted in some authors appearing who would not normally be considered leaders in tobacco control. They were primarily researchers working in epidemiology-often multi-risk factor epidemiology-whose work involved them in looking at the relation of smoking to disease. There were very few whose work involved tobacco control. Similarly, the 50 most cited papers were also dominated by epidemiological studies. The lists were decidedly narrow in showing the breadth of research scholarship examining all aspects of tobacco control policy, programmes, and the science underpinning these.

Throughout my own career, I have often noted papers that struck me as in some way seminal or as having made a research contribution that changed the ways in which our field thought about strategy and what needed to be done. I thought a parallel exercise where we invited people to vote for what they considered, simply, are the most important and influential papers in tobacco control might produce an interesting list.

\section{METHOD}

Over one month, all corresponding authors on papers published in Tobacco Control since 2001, as well as the members of the journal's editorial advisory board and its senior editors, were invited to go to a closed website to nominate up to five papers in each of 12 broad subject categories which they regarded as the "most important and influential papers" on tobacco control. Fifteen author's emails were returned as non-functional, leaving 202 who were invited to nominate. The software associated with the nomination process allowed the PubMed database to be searched and the unique identifying number of each nominated paper to be extracted to a database. Nominators could not see papers nominated by other nominators.

The 12 lists of papers thus obtained were then placed on a public website and thrown open for public voting for four weeks. Publicity about the voting was placed on the Tobacco Control homepage, on my own website, ${ }^{2}$ and via the Globalink and Society for Research on Nicotine and Tobacco membership list servers. The software limited each person's voting to five votes per category. Voters were unable to see the progressive voting totals as they voted. Papers nominated in more than one category were allowed to attract votes in each of their nominated categories.

\section{RESULTS}

The nomination phase saw 49 people nominate 658 different papers; 49 (19.8\%) made at least one nomination (range 160, mean 22.1). The voting phase saw 179 people vote 2966 times for these 658 papers. Papers listed by the nominators were included in the total votes. The 12 lists (tables 1-12) are shown below in order of the number of votes received for the 10 top papers in each category (in two categories there were 11 papers because of tied votes), as well as their citations as shown on the Institute of Scientific Information's Web of Science site as of June 2005.

Over three quarters of the leading papers were published in five journals: Tobacco Control (40), JAMA (20), BMJ (17), American Journal of Public Health (10), and New England Journal of Medicine (6).

\section{DISCUSSION}

Self nominations in the nomination phase were common although, with a few exceptions, by no means dominated any individual's list. It is possible that some authors may have urged their colleagues to vote for their papers, but if this occurred, it did not appear to be obvious in the pattern of voting. Voters had no way of knowing how many votes would have been needed to get them "over the line" into the top 10 in any category.

The average number of years since publication of papers in all categories was 8.5 years. In some categories (industry conduct, mass media, and "other") recent papers dominated the lists, suggesting a recent recall bias may have been operating or, in the case of industry conduct, the avalanche of recent work engendered by the availability of internal industry documents. It may have been that some considered "influence and importance" to mean importance to today's policy environment.

The lists of papers may be useful to teachers wishing to point students to a range of reading that those working in the field regard as important. Journals sometimes ask a prominent researcher to list important papers they would advise all newcomers to a field to read. This exercise has advanced the spirit of those sorts of lists further by engaging far more in the voting process.

Competing interests: none declared

Correspondence to: Professor Simon Chapman, School of Public Health, Edward Ford building A27, University of Sydney, NSW 2006, Australia; simonchapman@health.usyd.edu.au

\section{REFERENCES}

1 Byrne F, Chapman S. The most cited authors and papers in tobacco control. Tobacco Control 2005:14:155-60.

2 Tobacco Control Supersite. http://tobacco.health.usyd.edu.au/. 
Table 1 Secondhand smoke: 378 votes for 85 nominations. Top 10 received $48.6 \%$ of votes

\begin{tabular}{|c|c|c|}
\hline Paper & Votes & Citations \\
\hline $\begin{array}{l}\text { Hirayama T. Non-smoking wives of heavy smokers } \\
\text { have a higher risk of lung cancer: a study from } \\
\text { Japan. BMJ 1981;282:183-5. }\end{array}$ & 52 & 420 \\
\hline $\begin{array}{l}\text { Glantz SA, Parmley WW. Passive smoking and } \\
\text { heart disease. Mechanisms and risk. JAMA } \\
\text { 1995;273:1047-53. }\end{array}$ & 26 & 157 \\
\hline $\begin{array}{l}\text { Sargent RP, Shepard RM, Glantz SA. Reduced } \\
\text { incidence of admissions for myocardial infarction } \\
\text { associated with public smoking ban: before and } \\
\text { after study. BMJ 2004;328:977-80. }\end{array}$ & 22 & 7 \\
\hline $\begin{array}{l}\text { Repace JL, Lowrey AH. Indoor air pollution, tobacco } \\
\text { smoke, and public health. Science 1980;208:464-72. }\end{array}$ & 17 & 186 \\
\hline $\begin{array}{l}\text { Muggli ME, Hurt RD, Blanke DD. Science for hire: } \\
\text { a tobacco industry strategy to influence public } \\
\text { opinion on secondhand smoke. Nicotine Tob Res } \\
2003 ; 5: 303-14 \text {. }\end{array}$ & 16 & 24 \\
\hline $\begin{array}{l}\text { Barnes DE, Hanauer P, Slade J, Bero LA, Glantz SA. } \\
\text { Environmental tobacco smoke. The Brown and } \\
\text { Williamson documents. JAMA 1995;274:248-53. }\end{array}$ & 15 & 38 \\
\hline $\begin{array}{l}\text { Whincup PH, Gilg JA, Emberson JR, Jarvis MJ, } \\
\text { Feyerabend C, Bryant A, Walker M, Cook DG. } \\
\text { Passive smoking and risk of coronary heart disease } \\
\text { and stroke: prospective study with cotinine } \\
\text { measurement. BMJ 2004;329:200-5. }\end{array}$ & 15 & 6 \\
\hline $\begin{array}{l}\text { Scollo M, Lal A, Hyland A, Glantz S. Review of the } \\
\text { quality of studies on the economic effects of } \\
\text { smoke-free policies on the hospitality industry. } \\
\text { Tobacco Control 2003;12:13-20. }\end{array}$ & 11 & 21 \\
\hline $\begin{array}{l}\text { Glantz SA, Parmley WW. Passive smoking and } \\
\text { heart disease. Epidemiology, physiology, and } \\
\text { biochemistry. Circulation 1991;83:1-12. }\end{array}$ & 11 & 276 \\
\hline $\begin{array}{l}\text { Chapman S, Borland R, Scollo M, Brownson RC, } \\
\text { Dominello A, Woodward S. The impact of } \\
\text { smoke-free workplaces on declining cigarette } \\
\text { consumption in Australia and the United States. } \\
\text { Am J Public Health 1999;89:1018-23. }\end{array}$ & 10 & 53 \\
\hline
\end{tabular}

Table 2 Epidemiology of tobacco caused disease: 340 votes for 45 nominations. Top 10 received $50.3 \%$ of votes

\begin{tabular}{|c|c|c|}
\hline Paper & Votes & Citations \\
\hline $\begin{array}{l}\text { Doll } \mathrm{R} \text {, Peto R, Boreham J, Sutherland I. Mortality in } \\
\text { relation to smoking: } 50 \text { years' observations on male } \\
\text { British doctors. BMJ 2004;328:1519. }\end{array}$ & 42 & 22 \\
\hline $\begin{array}{l}\text { Hirayama T. Non-smoking wives of heavy smokers } \\
\text { have a higher risk of lung cancer: a study from } \\
\text { Japan. BMJ } 1981 ; 282: 183-5 \text {. }\end{array}$ & 24 & 420 \\
\hline $\begin{array}{l}\text { Wynder EL, Graham EA. Tobacco smoking as a } \\
\text { possible etiologic factor in bronchiogenic } \\
\text { carcinoma; a study of } 684 \text { proved cases. JAMA } \\
\text { 1950;143:329-36. }\end{array}$ & 18 & 538 \\
\hline $\begin{array}{l}\text { Peto R, Lopez AD, Boreham J, Thun M, Heath C. } \\
\text { Mortality from tobacco in developed countries: } \\
\text { indirect estimation from national vital statistics. } \\
\text { Lancet } 1992 ; 339: 1268-78 \text {. }\end{array}$ & 16 & 464 \\
\hline $\begin{array}{l}\text { Doll R, Peto R, Wheatley K, Gray R, Sutherland I. } \\
\text { Mortality in relation to smoking: } 40 \text { years' } \\
\text { observations on male British doctors. BMJ } \\
\text { 1994;309:901-11. }\end{array}$ & 13 & 736 \\
\hline $\begin{array}{l}\text { Doll R, Hill AB. A study of the aetiology of carcinoma } \\
\text { of the lung. BMU 1952;2:1271-86. }\end{array}$ & 13 & 389 \\
\hline $\begin{array}{l}\text { Doll } \mathrm{R} \text {, Hill } A B \text {. The mortality of doctors in relation } \\
\text { to their smoking habits: a preliminary report. } \\
\text { 1954. BMJ 1954:2;1451-5. }\end{array}$ & 12 & - \\
\hline $\begin{array}{l}\text { Glantz SA, Parmley WW. Passive smoking and } \\
\text { heart disease. Mechanisms and risk. JAMA } \\
\text { 1995;273:1047-53. }\end{array}$ & 11 & 157 \\
\hline $\begin{array}{l}\text { Ezzati } M \text {, Lopez AD. Estimates of global mortality } \\
\text { attributable to smoking in } 2000 \text {. Lancet } \\
2003 ; 362: 847-52 \text {. }\end{array}$ & 11 & 35 \\
\hline $\begin{array}{l}\text { Doll R, Hill AB. Smoking and carcinoma of the } \\
\text { lung; preliminary report. BMJ 1950;2:739-48. }\end{array}$ & 11 & - \\
\hline
\end{tabular}

Table 3 Cessation: 305 votes for 64 nominations. Top 10 received $44.9 \%$ of votes

\begin{tabular}{|c|c|c|}
\hline Paper & Votes & Citations \\
\hline $\begin{array}{l}\text { Novello AC. Surgeon General's report on the } \\
\text { health benefits of smoking cessation. Public Health } \\
\text { Rep 1990;105:545-8.* }\end{array}$ & 18 & 8 \\
\hline $\begin{array}{l}\text { Prochaska JO, DiClemente CC. Stages and } \\
\text { processes of self-change of smoking: toward an } \\
\text { integrative model of change. J Consult Clin Psychol } \\
1983 ; 51: 390-5 \text {. }\end{array}$ & 17 & 1347 \\
\hline $\begin{array}{l}\text { Novotny TE, Pierce JP, Giovino GA, et al. Methods } \\
\text { used to quit smoking in the United States. Do } \\
\text { cessation programs help? JAMA 1990;263:2760-5. }\end{array}$ & 17 & 289 \\
\hline $\begin{array}{l}\text { Russell MA, Wilson C, Taylor C, Baker CD. Effect } \\
\text { of general practitioners' advice against smoking. } \\
\text { BMJ 1979;2:231-5. }\end{array}$ & 17 & 565 \\
\hline $\begin{array}{l}\text { Fiore MC. US public health service clinical practice } \\
\text { guideline: treating tobacco use and dependence. } \\
\text { Respir Care 2000;45:1200-62. }\end{array}$ & 15 & \\
\hline $\begin{array}{l}\text { Zhu SH, Stretch V, Balabanis M, Rosbrook B, } \\
\text { Sadler G, Pierce JP. Telephone counseling for } \\
\text { smoking cessation: effects of single-session and } \\
\text { multiple-session interventions. J Consult Clin Psychol } \\
\text { 1996;64:202-11. }\end{array}$ & 12 & 85 \\
\hline $\begin{array}{l}\text { Jorenby DE, Leischow SJ, Nides MA et al. A } \\
\text { controlled trial of sustained-release bupropion, } \\
\text { a nicotine patch, or both for smoking cessation. } \\
\text { N Engl J Med 1999;340:685-91. }\end{array}$ & 12 & 436 \\
\hline $\begin{array}{l}\text { Doll R, Peto R, Boreham J, Sutherland I. Mortality } \\
\text { in relation to smoking: } 50 \text { years' observations on } \\
\text { male British doctors. BMJ 2004;328:1519. }\end{array}$ & 11 & 22 \\
\hline $\begin{array}{l}\text { Silagy C, Lancaster T, Stead L, Mant D, Fowler G. } \\
\text { Nicotine replacement therapy for smoking cessation. } \\
\text { Cochrane database of systematic reviews Cochrane } \\
\text { Database Syst Rev 2004;(3):CD000146. }\end{array}$ & 9 & - \\
\hline $\begin{array}{l}\text { Pierce JP, Farkas AJ, Gilpin EA. Beyond stages of } \\
\text { change: the quitting continuum measures progress } \\
\text { towards successful smoking cessation. Addiction } \\
1998 ; 93: 277-86 \text {. }\end{array}$ & 9 & 24 \\
\hline
\end{tabular}

Table 4 Youth: 254 votes for 60 nominations. Top 10 received $46.5 \%$ of votes

\begin{tabular}{|c|c|c|}
\hline Paper & Votes & Citations \\
\hline $\begin{array}{l}\text { Ling PM, Landman A, Glantz SA. It is time to abandon } \\
\text { youth access tobacco programmes. Tobacco Control } \\
2002 ; 11: 3-6 \text {. }\end{array}$ & 20 & 18 \\
\hline $\begin{array}{l}\text { Pierce JP, Choi WS, Gilpin EA, Farkas AJ, Merritt RK. } \\
\text { Validation of susceptibility as a predictor of which } \\
\text { adolescents take up smoking in the United States. } \\
\text { Health Psychol 1996;15:355-61. }\end{array}$ & 15 & 128 \\
\hline $\begin{array}{l}\text { DiFranza JR, Savageau JA, Rigotti NA, et al. } \\
\text { Development of symptoms of tobacco dependence in } \\
\text { youths: } 30 \text { month follow up data from the DANDY } \\
\text { study. Tobacco Control } 2002 ; 11: 228-35 \text {. }\end{array}$ & 13 & 42 \\
\hline $\begin{array}{l}\text { Lantz PM, Jacobson PD, Warner KE et al. Investing in } \\
\text { youth tobacco control: a review of smoking prevention } \\
\text { and control strategies. Tobacco Control } 2000 ; 9: 47-63 \text {. }\end{array}$ & 12 & 71 \\
\hline $\begin{array}{l}\text { Wayne GF, Connolly GN. How cigarette design can } \\
\text { affect youth initiation into smoking: Camel cigarettes } \\
\text { 1983-93. Tobacco Control 2002;1 1 (suppl I):i32-9. }\end{array}$ & 12 & 9 \\
\hline $\begin{array}{l}\text { Peterson AV, Kealey KA, Mann SL, Marek PM, } \\
\text { Sarason IG. Hutchinson smoking prevention project: } \\
\text { long-term randomized trial in school-based tobacco } \\
\text { use prevention-results on smoking. J Natl Cancer Inst } \\
\text { 2000;92:1979-91. }\end{array}$ & 10 & 86 \\
\hline $\begin{array}{l}\text { Ling PM, Glantz SA. Why and how the tobacco industry } \\
\text { sells cigarettes to young adults: evidence from industry } \\
\text { documents. Am J Public Health 2002;92:908-1. }\end{array}$ & 9 & 34 \\
\hline $\begin{array}{l}\text { Difranza JR, Tye JB. Who profits from tobacco sales to } \\
\text { children? JAMA 1990;63:2784-7. }\end{array}$ & 9 & 53 \\
\hline $\begin{array}{l}\text { Pollay RW. Targeting youth and concerned smokers: } \\
\text { evidence from Canadian tobacco industry documents. } \\
\text { Tobacco Control 2000;9:136-47. }\end{array}$ & 9 & 35 \\
\hline $\begin{array}{l}\text { DiFranza JR, Dussault GF. The federal initiative to halt the } \\
\text { sale of tobacco to children - the Synar Amendment, 1992- } \\
\text { 2000: lessons learned. Tobacco Control 2005;14:93-8. }\end{array}$ & 9 & 0 \\
\hline
\end{tabular}


Table 5 Epidemiology of tobacco use, knowledge, beliefs and attitudes: 251 votes for 45 nominations. Top 10 received $54.2 \%$ of votes

\begin{tabular}{|c|c|c|}
\hline Paper & Votes & Citations \\
\hline $\begin{array}{l}\text { Shiffman S, Pillitteri JL, Burton SL, Rohay JM, } \\
\text { Gitchell JG. Smokers' beliefs about "Light" and } \\
\text { "Ultra Light" cigarettes. Tobacco Control } \\
\text { 2001;10(suppl I):i17-23. }\end{array}$ & 22 & 18 \\
\hline $\begin{array}{l}\text { Giovino GA. Epidemiology of tobacco use } \\
\text { among US adolescents. Nicotine Tob Res } \\
1999 ; 1 \text { (suppl 1):S31-40. }\end{array}$ & 18 & - \\
\hline $\begin{array}{l}\text { Foulds J, Ramstrom L, Burke M, Fagerstróm K. } \\
\text { Effect of smokeless tobacco (snus) on smoking } \\
\text { and public health in Sweden. Tobacco Control } \\
\text { 2003;12:349-59. }\end{array}$ & 15 & 14 \\
\hline $\begin{array}{l}\text { Cummings KM, Morley CP, Hyland A. Failed } \\
\text { promises of the cigarette industry and its effect } \\
\text { on consumer misperceptions about the health } \\
\text { risks of smoking. Tobacco Control } \\
2002 ; 11 \text { (suppl I):i1 10-17. }\end{array}$ & 14 & 6 \\
\hline $\begin{array}{l}\text { Hammond D, Fong GT, McDonald PW, } \\
\text { Cameron R, Brown KS. Impact of the graphic } \\
\text { Canadian warning labels on adult smoking } \\
\text { behaviour. Tobacco Control 2003;12:391-5. }\end{array}$ & 13 & 2 \\
\hline $\begin{array}{l}\text { Lasser K, Boyd JW, Woolhandler S, } \\
\text { Himmelstein DU, McCormick D, Bor DH. Smoking } \\
\text { and mental illness: A population-based prevalence } \\
\text { study. JAMA 2000;284:2606-10. }\end{array}$ & 11 & 121 \\
\hline $\begin{array}{l}\text { Kozlowski LT, Rickert WS, Robinson JC, } \\
\text { Grunberg NE. Have tar and nicotine yields of } \\
\text { cigarettes changed? Science 1980;209:1550-1. }\end{array}$ & 11 & 34 \\
\hline $\begin{array}{l}\text { Niederdeppe J, Farrelly MC, Haviland ML. } \\
\text { Confirming "truth": more evidence of a successful } \\
\text { tobacco countermarketing campaign in Florida. } \\
\text { Am J Public Health 2004;94:255-7. }\end{array}$ & 11 & 3 \\
\hline $\begin{array}{l}\text { Weinstein ND, Marcus SE, Moser RP. Smokers' } \\
\text { unrealistic optimism about their risk. } \\
\text { Tobacco Control } 2005 ; 14: 55-9 .\end{array}$ & 11 & 0 \\
\hline $\begin{array}{l}\text { Pierce JP, Fiore MC, Novotny TE, Hatziandreu EJ, } \\
\text { Davis RM. Trends in cigarette smoking in the } \\
\text { United States. Projections to the year 2000. JAMA } \\
1989 ; 261: 61-5 \text {. }\end{array}$ & 10 & 280 \\
\hline
\end{tabular}

Table 6 Tobacco industry conduct: 243 votes for 68 nominations. Top 10 received $58 \%$ of votes

\begin{tabular}{|c|c|c|}
\hline Paper & Votes & Citations \\
\hline $\begin{array}{l}\text { Glantz SA, Barnes DE, Bero L, Hanauer P, Slade J. } \\
\text { Looking through a keyhole at the tobacco industry. } \\
\text { The Brown and Williamson documents. JAMA } \\
1995 ; 274: 219-24 \text {. }\end{array}$ & 34 & 51 \\
\hline $\begin{array}{l}\text { Slade J, Bero LA, Hanauer P, Barnes DE, Glantz SA. } \\
\text { Nicotine and addiction. The Brown and Williamson } \\
\text { documents. JAMA 1995;274:225-33. }\end{array}$ & 17 & 40 \\
\hline $\begin{array}{l}\text { Bero L. Implications of the tobacco industry } \\
\text { documents for public health and policy. Ann Rev } \\
\text { Public Health 2003;24:267-88. }\end{array}$ & 16 & 18 \\
\hline $\begin{array}{l}\text { Dearlove JV, Bialous SA, Glantz SA. Tobacco } \\
\text { industry manipulation of the hospitality industry } \\
\text { to maintain smoking in public places. Tobacco } \\
\text { Control 2002;11:94-104. }\end{array}$ & 11 & 29 \\
\hline $\begin{array}{l}\text { Hurt RD, Robertson CR. Prying open the door to } \\
\text { the tobacco industry's secrets about nicotine: the } \\
\text { Minnesota tobacco trial. JAMA 1998;280:1173-81. }\end{array}$ & 9 & 52 \\
\hline $\begin{array}{l}\text { Collin J, Legresley E, MacKenzie R, Lawrence S, } \\
\text { Lee K. Complicity in contraband: British American } \\
\text { Tobacco and cigarette smuggling in Asia. Tobacco } \\
\text { Control 2004;13(suppl II):ii104-11. }\end{array}$ & 9 & 3 \\
\hline $\begin{array}{l}\text { Francey N, Chapman S. "Operation Berkshire": } \\
\text { the international tobacco companies' conspiracy. } \\
\text { BMJ 2000;321:371-4. }\end{array}$ & 9 & 14 \\
\hline $\begin{array}{l}\text { Cummings KM, Morley CP, Hyland A. Failed } \\
\text { promises of the cigarette industry and its effect on } \\
\text { consumer misperceptions about the health risks of } \\
\text { smoking. Tobacco Control 2002;11(suppl I):i1 10-7. }\end{array}$ & 9 & 6 \\
\hline $\begin{array}{l}\text { Drope J, Chapman S. industry efforts at discrediting } \\
\text { scientific knowledge of environmental tobacco smoke: } \\
\text { a review of internal industry documents. J Epidemiol } \\
\text { Community Health } 2001 ; 55: 588-94 \text {. }\end{array}$ & 9 & 24 \\
\hline $\begin{array}{l}\text { Landman A, Ling PM, Glantz SA. Tobacco industry } \\
\text { youth smoking prevention programs: protecting the } \\
\text { industry and hurting tobacco control. Am J Public } \\
\text { Health 2002;92:917-30. }\end{array}$ & 9 & 10 \\
\hline $\begin{array}{l}\text { Barnes DE, Bero LA. Industry-funded research and } \\
\text { conflict of interest: an analysis of research sponsored } \\
\text { by the tobacco industry through the Center for } \\
\text { Indoor Air Research. J Health Polit Policy Law } \\
\text { 2002;92:917-30. }\end{array}$ & 9 & 44 \\
\hline
\end{tabular}


Table 7 Economics: 239 votes for 58 nominations. Top 10 received $54.8 \%$ of votes

\begin{tabular}{|c|c|c|}
\hline Paper & Votes & Citations \\
\hline $\begin{array}{l}\text { Warner KE. The economics of tobacco: myths and } \\
\text { realities. Tobacco Control 2000;9:78-89. }\end{array}$ & 31 & 16 \\
\hline $\begin{array}{l}\text { Scollo M, Lal A, Hyland A, Glantz S. Review of the } \\
\text { quality of studies on the economic effects of } \\
\text { smoke-free policies on the hospitality industry. } \\
\text { Tobacco Control 2003;12:13-20. }\end{array}$ & 19 & 21 \\
\hline $\begin{array}{l}\text { Chaloupka FJ, Cummings KM, Morley CP, } \\
\text { Horan JK. Tax, price and cigarette smoking: } \\
\text { evidence from the tobacco documents and } \\
\text { implications for tobacco company marketing } \\
\text { strategies. Tobacco Control 2002;11(suppl I):i62-72. }\end{array}$ & 19 & 5 \\
\hline $\begin{array}{l}\text { Efroymson D, Ahmed S, Townsend J et al. Hungry } \\
\text { for tobacco: an analysis of the economic impact of } \\
\text { tobacco consumption on the poor in Bangladesh. } \\
\text { Tobacco Control 2001;10:212-7. }\end{array}$ & 12 & 10 \\
\hline $\begin{array}{l}\text { Rice DP, Hodgson TA, Sinsheimer P, Browner W, } \\
\text { Kopstein AN. The economic costs of the health effects } \\
\text { of smoking, 1984. Milbank Quart 1986;64:489-547. }\end{array}$ & 11 & 81 \\
\hline $\begin{array}{l}\text { Warner KE, Fulton GA. The economic implications } \\
\text { of tobacco product sales in a nontobacco state. } \\
\text { JAMA 1994;271:771-6. }\end{array}$ & 10 & 13 \\
\hline $\begin{array}{l}\text { Jha P, Chaloupka FJ. The economics of global } \\
\text { tobacco control. BMJ 2000;321:358-61. }\end{array}$ & 10 & 32 \\
\hline $\begin{array}{l}\text { Curry SJ, Grothaus LC, McAfee T, Pabiniak C. Use } \\
\text { and cost effectiveness of smoking-cessation services } \\
\text { under four insurance plans in a health maintenance } \\
\text { organization. N Engl J Med 1998;339:673-9. }\end{array}$ & 10 & 89 \\
\hline $\begin{array}{l}\text { Warner KE, Hodgson TA, Carroll CE. Medical } \\
\text { costs of smoking in the United States: estimates, } \\
\text { their validity, and their implications. Tobacco Control } \\
1999 ; 8: 290-300 \text {. }\end{array}$ & 9 & 24 \\
\hline $\begin{array}{l}\text { Max W, Rice DP, Sung H-Y, Zhang X, Miller L. } \\
\text { The economic burden of smoking in California. } \\
\text { Tobacco Control 2004;13:264-7. }\end{array}$ & 9 & 0 \\
\hline
\end{tabular}

Table 8 Policy analysis, advocacy, legislation and litigation: 238 votes for 57 nominations. Top 10 received $47.9 \%$ of votes

\begin{tabular}{|c|c|c|}
\hline Paper & Votes & Citations \\
\hline $\begin{array}{l}\text { Barnes DE, Bero LA. Why review articles on the } \\
\text { health effects of passive smoking reach different } \\
\text { conclusions. JAMA 1998;279:1566-70. }\end{array}$ & 14 & 102 \\
\hline $\begin{array}{l}\text { Chapman S. Advocacy for public health: a primer. } \\
\text { J Epidemiol Community Health 2004;58:361-5. }\end{array}$ & 13 & 0 \\
\hline $\begin{array}{l}\text { Henningfield JE, Benowitz NL, Connolly GN et al. } \\
\text { Reducing tobacco addiction through tobacco product } \\
\text { regulation. Tobacco Control 2004;13:132-5. }\end{array}$ & 12 & 4 \\
\hline $\begin{array}{l}\text { Barnes DE, Hanauer P, Slade J, Bero LA, Glantz SA. } \\
\text { Environmental tobacco smoke. The Brown and } \\
\text { Williamson documents. JAMA 1995;274:248-53. }\end{array}$ & 12 & 38 \\
\hline $\begin{array}{l}\text { Wakefield M, Chaloupka F. Effectiveness of } \\
\text { comprehensive tobacco control programmes in } \\
\text { reducing teenage smoking in the USA. Tobacco } \\
\text { Control 2000;9:177-86. }\end{array}$ & 12 & 48 \\
\hline $\begin{array}{l}\text { Glantz SA, Parmley WW. Passive smoking and } \\
\text { heart disease. Mechanisms and risk. JAMA } \\
\text { 1995;273:1047-53. }\end{array}$ & 11 & 157 \\
\hline $\begin{array}{l}\text { Fichtenberg CM, Glantz SA. Effect of smoke-free } \\
\text { workplaces on smoking behaviour: systematic } \\
\text { review. BMJ 2002;325:188. }\end{array}$ & 11 & 62 \\
\hline $\begin{array}{l}\text { Borland R. A strategy for controlling the marketing } \\
\text { of tobacco products: a regulated market model. } \\
\text { Tobacco Control } 2003 ; 12: 374-82 \text {. }\end{array}$ & 11 & 4 \\
\hline $\begin{array}{l}\text { Chapman S. Unravelling gossamer with boxing } \\
\text { gloves: problems in explaining the decline in } \\
\text { smoking. BMJ 1993;307:429-32. }\end{array}$ & 9 & 32 \\
\hline $\begin{array}{l}\text { Fichtenberg CM, Glantz SA. Association of the } \\
\text { California Tobacco Control Program with declines } \\
\text { in cigarette consumption and mortality from heart } \\
\text { disease. N Engl J Med 2000;343:1772-7. }\end{array}$ & 9 & 63 \\
\hline
\end{tabular}

Table 9 Tobacco advertising, promotion, PR and packaging: 236 votes for 55 nominations. Top 10 received $47.0 \%$ of votes

\begin{tabular}{|c|c|c|}
\hline Paper & Votes & Citations \\
\hline $\begin{array}{l}\text { Fischer PM, Schwartz MP, Richards JW, } \\
\text { Goldstein AO, Rojas TH. Brand logo recognition } \\
\text { by children aged } 3 \text { to } 6 \text { years. Mickey Mouse and } \\
\text { Old Joe the Camel. JAMA 1991;266:3145-8. }\end{array}$ & 22 & 136 \\
\hline $\begin{array}{l}\text { Pierce JP, Lee L, Gilpin EA. Smoking initiation by } \\
\text { adolescent girls, } 1944 \text { through 1988. An } \\
\text { association with targeted advertising. JAMA } \\
\text { 1994;271:608-11. }\end{array}$ & 16 & 98 \\
\hline $\begin{array}{l}\text { Pierce JP, Gilpin E, Burns DM, et al. Does tobacco } \\
\text { advertising target young people to start smoking? } \\
\text { Evidence from California. JAMA 1991;266: } \\
\text { 3154-8. }\end{array}$ & 16 & 146 \\
\hline $\begin{array}{l}\text { DiFranza JR, Richards JW, Paulman PM, et al. } \\
\text { RJR Nabisco's cartoon camel promotes camel } \\
\text { cigarettes to children. JAMA 1991;266:3149-53. }\end{array}$ & 13 & 147 \\
\hline $\begin{array}{l}\text { Dalton MA, Sargent JD, Beach ML, et al. Effect of } \\
\text { viewing smoking in movies on adolescent smoking } \\
\text { initiation: a cohort study. Lancet } 2003 ; 362: 281-5 \text {. }\end{array}$ & 13 & 14 \\
\hline $\begin{array}{l}\text { Pollay RW, Dewhirst T. The dark side of marketing } \\
\text { seemingly "Light" cigarettes: successful images } \\
\text { and failed fact. Tobacco Control 2002;1 1(suppl I): } \\
\text { i18-31. }\end{array}$ & 10 & 11 \\
\hline $\begin{array}{l}\text { Pierce JP, Choi WS, Gilpin EA, Farkas AJ, } \\
\text { Berry CC. Tobacco industry promotion of } \\
\text { cigarettes and adolescent smoking. JAMA } \\
1998 ; 279: 511-5 \text {. }\end{array}$ & 10 & 153 \\
\hline $\begin{array}{l}\text { Wakefield M, Morley C, Horan JK, Cummings KM. } \\
\text { The cigarette pack as image: new evidence from } \\
\text { tobacco industry documents. Tobacco Control } \\
2002 ; 11 \text { (suppl I):i73-80. }\end{array}$ & 9 & 2 \\
\hline $\begin{array}{l}\text { Glantz SA, Kacirk KW, McCulloch C. Back to the } \\
\text { future: Smoking in movies in } 2002 \text { compared with } \\
1950 \text { levels. Am J Public Health 2004;94:261-3. }\end{array}$ & 9 & 2 \\
\hline $\begin{array}{l}\text { Landman A, Ling PM, Glantz SA. Tobacco industry } \\
\text { youth smoking prevention programs: protecting the } \\
\text { industry and hurting tobacco control. Am J Public } \\
\text { Health 2002;92:917-30. }\end{array}$ & 9 & 10 \\
\hline
\end{tabular}


Table 10 Mass media campaigns: 176 votes for 35 nominations. Top 10 received $64.8 \%$ of votes

\begin{tabular}{|c|c|c|}
\hline Paper & Votes & Citation \\
\hline $\begin{array}{l}\text { Pierce JP, Gilpin EA, Emery SL et al. Has the } \\
\text { California tobacco control program reduced } \\
\text { smoking? JAMA 1998;280:893-9. }\end{array}$ & 20 & 78 \\
\hline $\begin{array}{l}\text { Pierce JP, Macaskill P, Hill D. Long-term } \\
\text { effectiveness of mass media led antismoking } \\
\text { campaigns in Australia. Am J Public Health } \\
1990 ; 80: 565-9 .\end{array}$ & 15 & 54 \\
\hline $\begin{array}{l}\text { Sly DF, Heald GR, Ray S. The Florida "truth" anti- } \\
\text { tobacco media evaluation: design, first year results, } \\
\text { and implications for planning future state media } \\
\text { evaluations. Tobacco Control } 2001 ; 10: 9-15 \text {. }\end{array}$ & 14 & 32 \\
\hline $\begin{array}{l}\text { Farrelly MC, Davis KC, Haviland ML, Messeri P, } \\
\text { Healton CG. Evidence of a dose-response } \\
\text { relationship between "truth" antismoking ads and } \\
\text { youth smoking prevalence. Am J Public Health } \\
2005 ; 95: 425-31 \text {. }\end{array}$ & 11 & 0 \\
\hline $\begin{array}{l}\text { Farrelly MC, Healton CG, Davis KC, Messeri P, } \\
\text { Hersey JC, Haviland ML. Getting to the truth: } \\
\text { evaluating national tobacco countermarketing } \\
\text { campaigns. Am J Public Health 2002;92:901-7. }\end{array}$ & 9 & 52 \\
\hline $\begin{array}{l}\text { Biener L, McCallum-Keeler G, Nyman AL. Adults' } \\
\text { response to Massachusetts anti-tobacco television } \\
\text { advertisements: impact of viewer and advertisement } \\
\text { characteristics. Tobacco Control 2000;9:401-7. }\end{array}$ & 8 & 11 \\
\hline $\begin{array}{l}\text { Borland R, Balmford J. Understanding how mass } \\
\text { media campaigns impact on smokers. Tobacco } \\
\text { Control 2003;12(suppl II):ii45-52. }\end{array}$ & 8 & 3 \\
\hline $\begin{array}{l}\text { Wakefield M, Flay B, Nichter M, Giovino G. } \\
\text { Effects of anti-smoking advertising on youth smoking: } \\
\text { a review. J Health Communication 2003;8:229-47. }\end{array}$ & 8 & 6 \\
\hline $\begin{array}{l}\text { Pierce JP, Gilpin EA. News media coverage of } \\
\text { smoking and health is associated with changes in } \\
\text { population rates of smoking cessation but not } \\
\text { initiation. Tobacco Control 2000;10:145-53. }\end{array}$ & 7 & 15 \\
\hline $\begin{array}{l}\text { Sargent JD, Beach ML, Dalton MA et al. Effect of } \\
\text { parental R-rated movie restriction on adolescent } \\
\text { smoking initiation: a prospective study. Pediatrics } \\
2004 ; 114: 149-56 \text {. }\end{array}$ & 7 & 1 \\
\hline $\begin{array}{l}\text { Warner KE, Jacobson PD, Kaufman NJ. Innovative } \\
\text { approaches to youth tobacco control: introduction } \\
\text { and overview. Tobacco Control 2003;12(suppl I): } \\
\text { i1-15. }\end{array}$ & 7 & 1 \\
\hline
\end{tabular}

Table 11 Pharmacology: 172 votes for 34 nominations. Top 10 received $58.7 \%$ of votes

\begin{tabular}{|c|c|c|}
\hline Paper & Votes & Citations \\
\hline $\begin{array}{l}\text { Benowitz NL, Hall SM, Herning RI, Jacob P, } \\
\text { Jones RT, Osman AL. Smokers of low-yield } \\
\text { cigarettes do not consume less nicotine. } \\
\text { N Engl J Med 1983;309:139-42. }\end{array}$ & 19 & 306 \\
\hline $\begin{array}{l}\text { Henningfield JE, Benowitz NL, Connolly GN, et al. } \\
\text { Reducing tobacco addiction through tobacco product } \\
\text { regulation. Tobacco Control 2004;13:132-5. }\end{array}$ & 14 & 4 \\
\hline $\begin{array}{l}\text { Bialous SA, Yach D. Whose standard is it, anyway? } \\
\text { How the tobacco industry determines the } \\
\text { International Organization for Standardization } \\
\text { (ISO) standards for tobacco and tobacco products. } \\
\text { Tobacco Control } 2001 ; 10: 96-104 \text {. }\end{array}$ & 12 & 20 \\
\hline $\begin{array}{l}\text { Jarvis MJ, Boreham R, Primatesta P, Feyerabend C, } \\
\text { Bryant A. Nicotine yield from machine-smoked } \\
\text { cigarettes and nicotine intakes in smokers: evidence } \\
\text { from a representative population survey. J Natl } \\
\text { Cancer Inst } 2001 ; 93: 134-8 \text {. }\end{array}$ & 12 & 39 \\
\hline $\begin{array}{l}\text { Kozlowski LT, Frecker RC, Khouw V, Pope MA. } \\
\text { The misuse of 'less-hazardous' cigarettes and its } \\
\text { detection: hole-blocking of ventilated filters. Am J } \\
\text { Public Health 1980;70:1202-3. }\end{array}$ & 11 & 72 \\
\hline $\begin{array}{l}\text { DiFranza JR, Savageau JA, Rigotti NA et al. } \\
\text { Development of symptoms of tobacco dependence in } \\
\text { youths: } 30 \text { month follow up data from the DANDY } \\
\text { study. Tobacco Control } 2002 ; 11: 228-35 \text {. }\end{array}$ & 7 & 42 \\
\hline $\begin{array}{l}\text { Benowitz NL, Jacob P, Kozlowski LT, Yu L. Influence } \\
\text { of smoking fewer cigarettes on exposure to tar, } \\
\text { nicotine, and carbon monoxide. N Engl J Med } \\
\text { 1986;315:1310-3. }\end{array}$ & 7 & 105 \\
\hline $\begin{array}{l}\text { Benowitz NL. Pharmacology of nicotine: addiction } \\
\text { and therapeutics. Ann Rev Pharmacol Toxicol } \\
\text { 1996;36:597-613. }\end{array}$ & 7 & 169 \\
\hline $\begin{array}{l}\text { Pontieri FE, Tanda G, Orzi F, Di Chiara G. Effects of } \\
\text { nicotine on the nucleus accumbens and similarity to } \\
\text { those of addictive drugs. Nature 1996;382:255-7. }\end{array}$ & 6 & 349 \\
\hline $\begin{array}{l}\text { Russell MA. Cigarette smoking: natural history of } \\
\text { a dependence disorder. Br J Med Psychol } \\
1971 ; 44: 1-16 \text {. }\end{array}$ & 6 & \\
\hline
\end{tabular}

Table 12 Other: 134 votes for 22 nominations. Top 10 received $78.4 \%$ of votes

\begin{tabular}{|c|c|c|}
\hline Paper & Votes & Citations \\
\hline $\begin{array}{l}\text { Fichtenberg CM, Glantz SA. Association of the } \\
\text { California Tobacco Control Program with declines } \\
\text { in cigarette consumption and mortality from heart } \\
\text { disease. N Engl J Med 2000;343:1772-7. }\end{array}$ & 14 & 63 \\
\hline $\begin{array}{l}\text { Efroymson D, Ahmed S, Townsend J, et al. Hungry } \\
\text { for tobacco: an analysis of the economic impact of } \\
\text { tobacco consumption on the poor in Bangladesh. } \\
\text { Tobacco Control 2001; 10:212-7. }\end{array}$ & 12 & 10 \\
\hline $\begin{array}{l}\text { Henningfield JE, Benowitz NL, Connolly GN, et al. } \\
\text { Reducing tobacco addiction through tobacco product } \\
\text { regulation. Tobacco Control 2004;13:132-5. }\end{array}$ & 12 & 4 \\
\hline $\begin{array}{l}\text { Bero LA, S Glantz. The limits of competing interest } \\
\text { disclosures. Tobacco Control 2005;14:118-26. }\end{array}$ & 12 & 0 \\
\hline $\begin{array}{l}\text { Mahood G. Warnings that tell the truth: breaking } \\
\text { new ground in Canada. Tobacco Control } \\
1999 ; 8: 356-61 \text {. }\end{array}$ & 12 & 9 \\
\hline $\begin{array}{l}\text { Ernster V, Kaufman N, Nichter M, Samet J, } \\
\text { Yoon SY. Women and tobacco: moving from policy } \\
\text { to action. Bull World Health Org 2000;78:891-901. }\end{array}$ & 10 & 23 \\
\hline $\begin{array}{l}\text { Chapman S. Advocacy in public health: roles and } \\
\text { challenges. Int J Epidemiol 2001;30:1226-32. }\end{array}$ & 9 & 8 \\
\hline $\begin{array}{l}\text { Chapman S. Unravelling gossamer with boxing } \\
\text { gloves: problems in explaining the decline in } \\
\text { smoking. BMJ 1993;307:429-32. }\end{array}$ & 8 & 32 \\
\hline $\begin{array}{l}\text { Joossens L, Raw M. How can cigarette smuggling } \\
\text { be reduced? BMJ 2000;321:947-50. }\end{array}$ & 8 & 17 \\
\hline $\begin{array}{l}\text { Shiffman S, Pillitteri JL, Burton SL, Di Marino ME. } \\
\text { Smoker and ex-smoker reactions to cigarettes claiming } \\
\text { reduced risk. Tobacco Control 2004;13:78-84. }\end{array}$ & 8 & 0 \\
\hline
\end{tabular}

